\title{
ISOLATED EIGENVALUES, POLES AND COMPACT PERTURBATIONS OF BANACH SPACE OPERATORS
}

\author{
BHAGWATI PRASHAD DUGGAL
}

Abstract. Given a Banach space operator $A$, the isolated eigenvalues $E(A)$ and the poles $\Pi(A)$ (resp., eigenvalues $E^{a}(A)$ which are isolated points of the approximate point spectrum and the left ploles $\Pi^{a}(A)$ ) of the spectrum of $A$ satisfy $\Pi(A) \subseteq E(A)$ (resp., $\Pi^{a}(A) \subseteq E^{a}(A)$ ), and the reverse inclusion holds if and only if $E(A)$ (resp., $E^{a}(A)$ ) has empty intersection with the B-Weyl spectrum (resp., upper B-Weyl spectrum) of $A$. Evidently $\Pi(A) \subseteq E^{a}(A)$, but no such inclusion exists for $E(A)$ and $\Pi^{a}(A)$. The study of identities $E(A)=\Pi^{a}(A)$ and $E^{a}(A)=\Pi(A)$, and their stability under perturbation by commuting Riesz operators, has been of some interest in the recent past. This paper studies the stability of these identities under perturbation by (noncommuting) compact operators. Examples of analytic Toeplitz operators and operators satisfying the abstract shift condition are considered.

Mathematics subject classification (2010): 47A10, 47A55, 47A53, 47B40.

Keywords and phrases: Banach space operator, isolated eigenvalues, left poles, poles, compact perturbations, SVEP, Fredholm operator, Toeplitz operator, abstract shift condition.

\section{REFERENCES}

[1] P. AIEnA, Fredholm and Local Spectral Theory with Applications to Multipliers, Kluwer, 2004.

[2] P. Aiena, J. Guillen And P. Pena, Property $(R)$ for bounded linear operators, Mediterr. J. Mat. 8(2011), 491-508.

[3] P. Aiena, E. Aponte, J. Guillen and P. Pena, Property $(R)$ under perturbations, Mediterr. J. Math. 10(2013), 367-382.

[4] P. Aiena, J. Guillen And P. Pena, Property $(g R)$ and perturbations, Acta Sci. Math.(Szeged) 78(2012), 569-588.

[5] B. P. Duggal, Dragan S. Djordjević, Robin E. Harte and Snežana C. ŽivkovićZlatanović, Polynomially meromorphic operators, Math. Proc. Royal Irish Acad. 116A(2016), 8398.

[6] B. P. DugGal And I. H. KIM, Generalized Browder, Weyl spectra and the polaroid property under compact perturbations, J. Korean Math. Soc. 54(2017), 281-302.

[7] B. P. Duggal, Polaroid operators and generalized Browder-Weyl theorems, Math. Proc. Royal Irish Acad. 108A(2008), 149-163.

[8] B. P. DugGal, Spectral picture, perturbed Browder and Weyl theorems, and their variations, Functional Analysis, Approximation and Computation 9(1)(2017), 1-23.

[9] B. P. Duggal, Perturbed Browder, Weyl theorems and their variations: Equivalences, Functional Analysis, Approximation and Computation 9(2)(2017), 37-62.

[10] P. R. Halmos, A Hilbert Space Problem Book, 2nd edition, Springer 1982.

[11] D. A. Herrero, T. J. TAYlor And Z. Y. WAng, Variation of the point spectrum under compact perturbations, Topics in Operator Theory: Operator Theory Advances and Application, Birkhäuser, Basel Volume 32(1988), 113-158.

[12] H. G. Heuser, Functional Analysis, John Wiley and Sons (1982).

[13] You QIng Ji, Quasitriagular + small compact = strongly irreducible, Trans. Amer. Math. Soc. 351(1999), 4657-4673. 
[14] K. B. Laursen And M. M. Neumann, Introduction to Local Spectral Theory, Clarendon Press, Oxford, 2000.

[15] C. G. Li AND T. T. Zhou, Polaroid type operators and compact perturbations, Studia Math. 221(2014), 175-192.

[16] V. MüLLER, Spectral Theory of Linear Operators and Spectral Systems in Banach Algebras, 2nd edition, Birkhauser, Basel 2007.

[17] M. H. RASHID, Properties $(S)$ and $(g S)$ for bounded linear operators, Filomat 28(2014), 16411652.

[18] Junli Shen And Alatancang CHen, A new variation on Weyl type theorems and perturbations, Filomat 28(2114), 1899-1906.

[19] Angus E. Taylor and David C. Lay, Introduction to Functional Analysis, John Wiley and Sons (1980).

[20] H. ZARiouh, Preservation results for new spectral properties, Extracta Math. 30(2015), 191-205.

[21] Sen Zhu And Chun GuAng Li, SVEP and compact perturbations, J. Math. Anal. Appl.,380(2011), 69-75. 